\title{
What is new in tics, dystonia and chorea?
}

\author{
Authors: Antonella Macerollo ${ }^{A}$ and Davide Martino ${ }^{B}$
}

Movement disorders comprise hyperkinetic involuntary movements (eg tremor, myoclonus, tics, dystonia and chorea) and hypokinetic (parkinsonism) disorders. Tics are cardinal features of primary tic disorders encompassing Tourette syndrome (TS), but are also found in some neurodegenerative conditions and may be induced by psychoactive substances. The first line treatment for tics is pharmacological (mainly dopamine receptor blockers or alpha-2 adrenergic agonists) and behavioural. Dystonia and chorea syndromes are considerably heterogeneous in aetiology, and age at onset, body distribution of the movement disorder, accompanying neurological motor and non-motor features, and systemic manifestations are all important to reach a correct aetiological diagnosis. While symptomatic pharmacological treatment remains the mainstay of treatment for choreas, deep brain stimulation surgery has a well-defined place in the management of medically refractory dystonia.

\section{Introduction}

Movement disorders comprise hyperkinetic involuntary movements (eg tremor, myoclonus, tics, dystonia and chorea) and hypokinetic (parkinsonism) disorders. ${ }^{1}$ Because of the complex aetiology of movement disorder syndromes, accurate phenomenological characterisation of the clinical features of these syndromes is an important first step to plan the correct diagnostic work-up and guide further management and referral to the appropriate neurology services.

Here, we provide a brief overview of three subtypes of hyperkinetic disorders (tics, dystonia and chorea), discussing their clinical diagnosis and therapeutic approach. Despite the lack of disease-modifying treatment for the majority of these neurological disorders, an effort to improve diagnostic acumen remains paramount for the provision of prognosis to the patients and their families, and for patient recruitment in future therapeutic trials.

Authors: ${ }^{\text {A }}$ clinical research associate, Institute of Neurology, University College of London, London, UK; ${ }^{\mathrm{B}}$ consultant neurologist, National Parkinson Foundation International Centre of Excellence, King's College Hospital NHS Trust, London, UK and Queen Elizabeth Hospital, Lewisham and Greenwich NHS Trust, London, UK
Tics

\section{Clinical presentation}

Tics are repetitive and patterned movements or phonic utterances that appear out of context, may be partially suppressed under volition, and are often preceded by uncomfortable urges that are temporarily relieved by tic production. Tics are the defining feature of neurodevelopmental chronic tic disorders, among which Tourette syndrome (TS) remains the most clinically relevant primary tic disorder.

\section{Key points}

Tourette syndrome exhibits an important comorbidity with attention-deficit/hyperactivity disorder or obsessive compulsive disorder ( $72 \%$ ), mood, anxiety and disruptive behaviour disorders (each approximately $30 \%$ ).

Several randomised controlled trials and meta-analyses support alpha-2 adrenergic agonists and dopamine receptor blockers as first line treatment for tics.

Dystonic movements and postures can occur at rest but are typically aggravated by voluntary movement and, in some forms of dystonia, is almost exclusively triggered by specific complex motor actions (eg writing dystonia, musician's dystonia).

Chemodenervation with botulinum neurotoxins is the most effective and widely available treatment for segmental/focal dystonias, whereas deep brain stimulation surgery has gained an important role in the management of medically refractory dystonia.

The different causes of chorea may be recognised based on course (acute/subacute or chronic) and body distribution (focal, segmental, unilateral, generalised).

The pharmacological treatment of chorea involves D2 dopamine receptor blockers, tetrabenazine and antiepileptic agents (mainly carbamazepine and valproic acid).

KEYWORDS: Chorea, diagnosis, dystonia, hyperkinetic movement disorders, tics, treatments 
According to the recent Diagnostic and Statistical Manual of Mental Disorders V (DSM-V) criteria, TS is diagnosed by the presence of motor and vocal (or phonic) tics, which manifest before the age of 18 for more than 12 months in the absence of secondary causes. ${ }^{2}$ Tics are less commonly secondary to other conditions or causative factors (Box 1$).^{3}$

In a meta-analysis of prevalence data from 1985 to 2011, studies applying a school-based assessment yielded a combined prevalence of $0.77 \%$ for TS, $1.61 \%$ for chronic tic disorders, 2.99\% for transient tic disorders, and 2.82\% for the 'all tic disorders' category in the population up to 16 years of age. ${ }^{4} 2015$ population-based data have confirmed that only about $15 \%$ of TS patients do not suffer from any other psychiatric comorbidity, with the majority of patients affected by attention-deficit/hyperactivity disorder (ADHD) or obsessive compulsive disorder (72\% cumulatively), or mood, anxiety and disruptive behaviour (impulsive behaviours, rage attacks) disorders (each approximately $30 \%) .{ }^{5}$ This striking comorbidity profile should be taken into account during the assessment of TS patients, in order to identify treatment priorities in each individual patient.

Tics are now viewed as the result of altered inhibitory control mechanisms modulating action selection. Importantly, tics are highly influenced by sensory, cognitive and emotional inputs, suggesting dysfunctional crosstalk between all three of the main cortico-basal ganglia circuits (motor, associative and limbic). Recent advances coming from neurophysiological, ${ }^{6}$ imaging (magnetic resonance imaging (MRI) and magnetoencephalography), ${ }^{7,8}$ neurobiological (animal models) ${ }^{9}$ and neuropathological studies confirmed the involvement of dysfunctional inhibitory control at the level of both inhibitory projection neurons or interneurons in the striatum and cortical microcircuits. A relationship has been found between vocal tics and abnormal connectivity of the ventral striatum within cortico-subcortical limbic circuits in a primate model. Importantly, post-mortem studies documented reduced numbers and function of striatal interneurons in adults with TS. ${ }^{10}$

\section{Treatment}

Therapeutic algorithms like those published by the European Society for the Study of Tourette Syndrome (Fig 1) identify three main decisional stages in the treatment of tics. After diagnosis and comprehensive assessment, a more active intervention for tics (ie beyond psychoeducation) is necessary only when tics impact on social and academic functioning, are stigmatising, or potentially physically harmful. ${ }^{11}$ Once a decision to treat tics more actively is taken, the next step is to decide between behavioural and pharmacological treatment.

A behavioural approach, like the Comprehensive Behavioural Intervention for Tics, which applies habit reversal training, is supported by high quality trials on youths and adults with TS. ${ }^{12}$ Other approaches, such as exposure response prevention and cognitive psychophysiological models, still require testing in higher quality trials, and the efficacy of their combination with pharmacological treatments remains to be investigated. Moreover, behavioural treatments may be difficult to apply in younger children (below age 10) or in patients with severe comorbid ADHD. ${ }^{13}$

\section{Box 1. Secondary causes of tics}

\section{Neurodevelopmental disorders}

Mental retardation

Autistic spectrum disorders (including Asperger syndrome)

Rett syndrome

Genetic and chromosomal abnormalities

X-linked mental retardation (MRX23)

Albright hereditary osteodystrophy

Duchenne muscular dystrophy

Factor VIII hemophilia

Fragile $X$ syndrome

Lesch-Nyhan syndrome

Triple $X$ and $9 p$ mosaicism

47 XXY karyotype

Partial trisomy 16

$9 p$ monosomy

Beckwith-Wiedemann syndrome

Tuberous sclerosis

$>$ Congenital adrenal hyperplasia due to 21-hydroxylase deficiency

Phenylketonuria

Corpus callosum dysgenesis

$>$ Craniosynostosis

Klinefelter syndrome

Neurofibromatosis

Developmental stuttering

\section{Acute brain lesions}

$>$ Post-traumatic

Vascular

Infectious

Varicella-zoster virus

Herpes simplex encephalitis

Mycoplasma pneumoniae

Lyme disease

\section{Post-infectious}

Sydenham's chorea

Pediatric autoimmune neuropsychiatric disorder associated with streptococcal infections (PANDAS)

\section{Neurodegenerative diseases}

$>$ Huntington disease

Neuroacanthocytosis syndromes

Neurodegeneration with brain iron accumulation

Other systemic diseases

Behçet's syndrome

Antiphospholipid syndrome

Peripheral trauma

Medications and toxins

Amphetamines, cocaine, heroin, methylphenidate, pemoline, antipsychotics (tardive tics), antidepressants, antiepileptics (carbamazepine, phenytoin, phenobarbital, lamotrigine), L-dopa

Functional tic-like jerks 


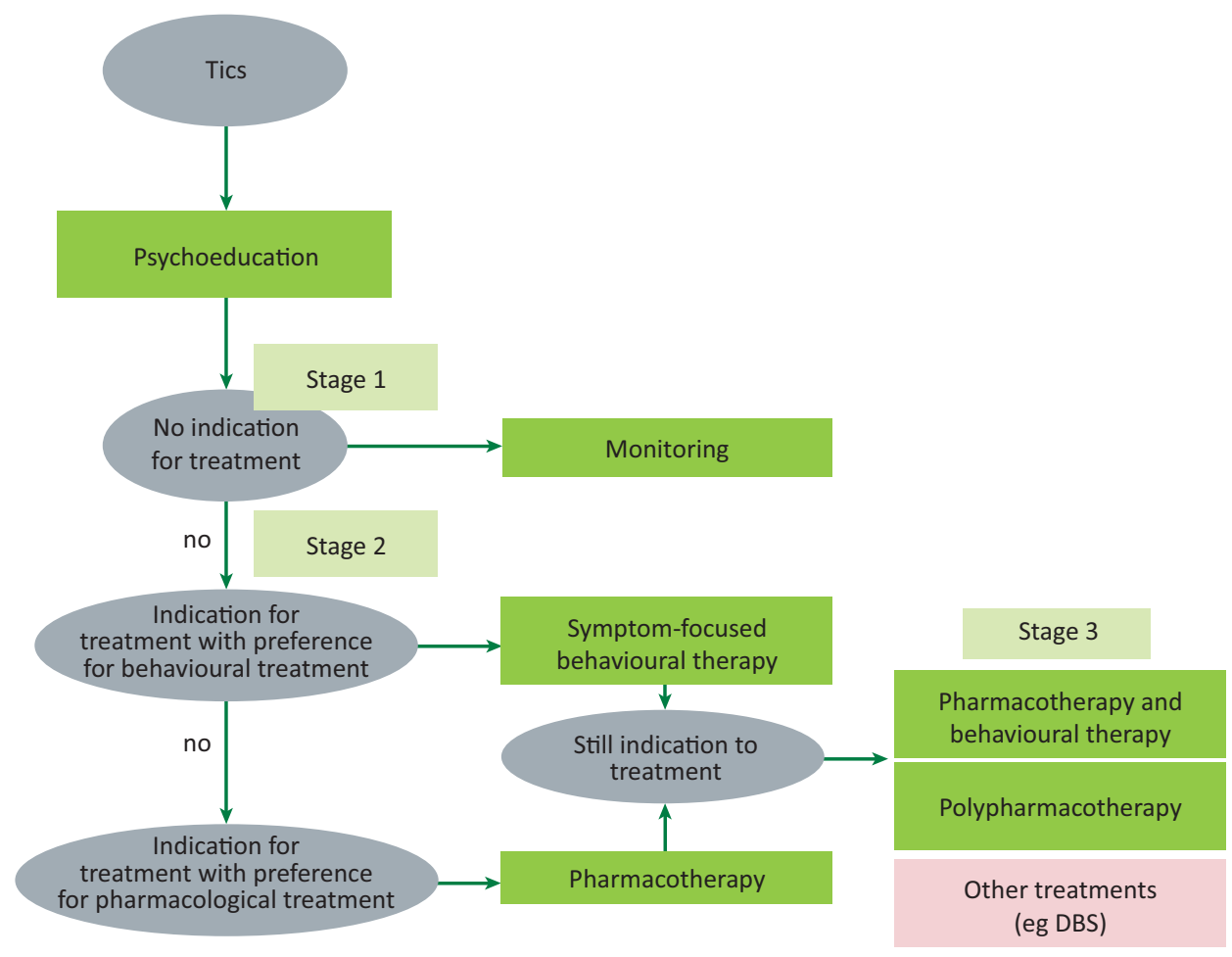

Fig 1. Therapeutic algorithms for treatment of tic disorders. Decision tree for the treatment of tics (specifically focused on Tourette syndrome and other primary tic disorders). ${ }^{11}$ DBS $=$ deep brain stimulation.

Several randomised controlled trials and meta-analyses support alpha-2 adrenergic agonists and dopamine receptor blockers as first line agents for the treatment of tics. The former group of drugs (guanfacine - unavailable in several European countries - and clonidine) appears to be effective against tics predominantly in TS patients with comorbid ADHD. ${ }^{14}$ Among dopamine receptor blockers, aripiprazole, risperidone, pimozide and haloperidol were confirmed to be effective in meta-analyses, although the poor tolerability of first generation antipsychotics (pimozide, haloperidol) strongly limits their routine use. ${ }^{15}$ Although not supported by a similar degree of evidence quality, fluphenazine and benzamides are often favoured because of their better tolerability profile than a non-antipsychotic dopamine depletor like tetrabenazine. Botulinum toxin may have great utility in cranial and cervical motor tics, as well as in vocal tics.

Patients with severe, highly disabling tics who are unresponsive to first line interventions are now more and more frequently considered for neuromodulation strategies, such as deep brain stimulation (DBS). More than 130 patients with TS have been treated with DBS worldwide, with less than a dozen cases under the age of 18 years. ${ }^{16}$ In patients under 18 years of age, DBS should be considered only in those whose psychiatric comorbidities are stable and who do not present with suicidality or high risk of aggressiveness directed against others. Although priority anatomical targets are still being debated, crossover sham-controlled trials support the efficacy of globus pallidus internus (limbic portion particularly) ${ }^{17}$ and centromedian/parafascicularis nuclei stimulation, ${ }^{18}$ with an acceptable short- and mediumterm adverse effect profile.

\section{Dystonia}

\section{Clinical presentation}

Dystonia is a movement disorder characterised by sustained muscle contractions, which frequently cause abnormal postures (tonic component) or repetitive movements (phasic component). The typical clinical features of dystonic contractions are marked directionality, long duration, and simultaneous involvement of agonist and antagonist muscles. ${ }^{19}$ Dystonic movements can less commonly be more rapid and have a rhythmic pattern, resembling a tremor. However, in most cases, dystonic tremor may show an evident directionality, unlike the typical sinusoidal oscillations of other forms of tremor. ${ }^{20}$

Dystonic movements and postures can occur at rest but are typically aggravated by voluntary movement. In some forms, dystonia is almost exclusively triggered by specific complex motor actions performed with the same body part (eg writing dystonia, musician's dystonia). Dystonic contractions within a specific body region can also be triggered by voluntary movements occurring at distant segments (overflow phenomena). ${ }^{21} \mathrm{~A}$ rare phenomenon is the suppression of dystonia during voluntary movements (paradoxical dystonia) and involves the facial muscles. ${ }^{19,21}$

Gestes antagonistes (also known as 'sensory tricks') are typical features of dystonic movements and indicate temporary reduction in the severity of dystonia by using an alternative motor action, which often conveys tactile or proprioceptive stimulation; typical gestes include touching the chin or the neck in cervical dystonia. ${ }^{22}$

The clinical classification of dystonia has been recently revised, and comprises two main axes: clinical characteristics and aetiology (Fig 2). ${ }^{23}$ 
Fig 2. Classification of dystonia. Dystonia is currently classified according to two axes: clinical characteristics and aetiology. Clinical characteristics include age at onset, body distribution, temporal pattern and associated features. The aetiological axis refers to presence of nervous system pathology and whether these are inherited, acquired or idiopathic in nature (from Martino D, Espay A], Fasano A, Morgante F. Disorders of movement: a guide to diagnosis and treatment. Heidelberg: Springer-Verlag, 2016). MDS = movement disorders.

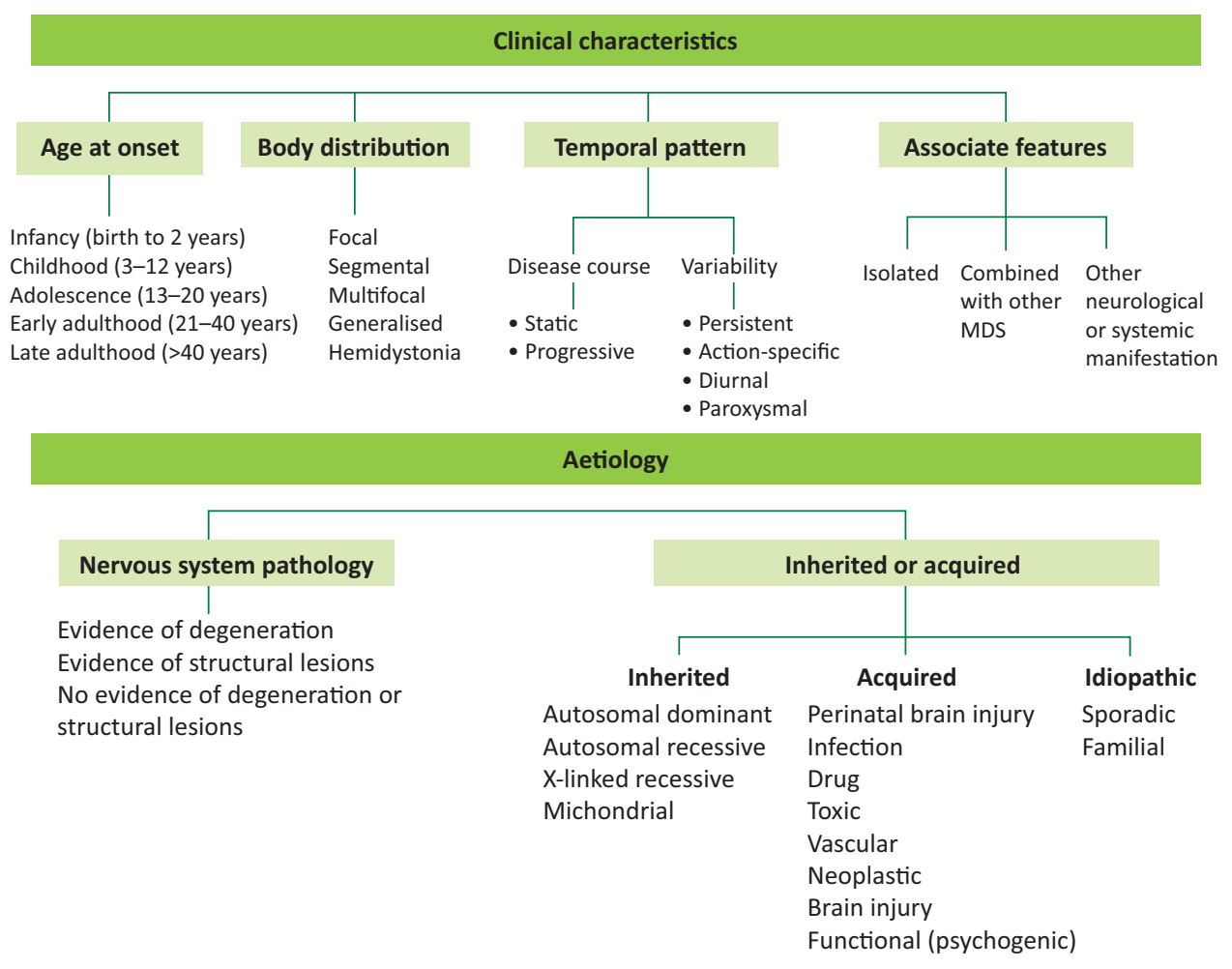

Isolated (formerly known as primary) dystonia encompasses all those conditions in which dystonia is the sole relevant clinical feature, with the exception of associated tremor. This is a genetically heterogeneous group of disorders. Early onset forms (ie age at onset $<26$ years) become frequently generalised and are associated with TOR1A (DYT1, without cranio-cervical involvement) and THAP1 (DYT6, with cranio-cervical involvement) gene mutations. More than five other potentially causative genes have been described in the last 3 years, which are also associated with adult-onset (focal) forms. However, their role in aetiology and diagnosis is still under investigation. ${ }^{24}$ Combined dystonia syndromes include myoclonus-dystonia syndromes (also genetically heterogeneous) and dystonia-parkinsonism syndromes; the latter group includes dopa-responsive forms and heredodegenerative diseases that include the complex family of neurodegeneration with brain iron accumulation disorders (Box 2).

\section{Treatment}

The mainstay treatment for isolated dystonia and the majority of secondary dystonias remains pharmacological, even though early DBS for monogenic forms of isolated generalised dystonia is now extensively applied. Anticholinergic drugs (eg trihexyphenidyl) remain first line agents for multifocal or generalised dystonias, and their dose should be titrated gradually to minimise side effects, particularly dry mouth, gastrointestinal upset, urinary retention and confusion. ${ }^{25}$ Muscle relaxants such as baclofen (available also in intrathecal pump infusion formulation) and clonazepam can be helpful when spasticity or tremor, respectively, are associated with dystonia; lethargy, dizziness and confusion may be associated with these drugs. Dopamine-depleting drugs such as tetrabenazine may be helpful in a smaller proportion of cases. L-dopa in small doses (50-200 mg) represents the first line treatment for dopa-responsive dystonias and, given the costs of genetic screening for these forms of dystonia, an empiric trial of L-dopa is usually offered to all patients with early onset dystonia in whom there is no evidence of neurodegeneration or brain structural lesions. ${ }^{26}$ Chemodenervation with botulinum neurotoxins (three forms of botulinum toxin $\mathrm{A}$ and one of botulinum toxin B are available in the UK) is a very effective and widely available treatment for segmental/ focal dystonias in which the topography of the muscles involved can be determined. ${ }^{27}$ Electromyographic and ultrasound guidance techniques have greatly advanced recently for those body regions, like upper and lower limbs, in which the superficial injection approach is hindered by the complexity of anatomical localisation of the muscles contributing to dystonia. ${ }^{25,28}$

DBS is considered for highly disabling, medically refractory dystonias. DBS of the globus pallidus internus (GPi) has been shown to be very effective for the treatment of DYT1 dystonia and, to a slightly lesser extent, for non-DYT1, early-onset generalised dystonias. A 35-45\% improvement in severity was also reported for refractory cervical dystonia treated with GPi DBS. There is a less quality evidence to support DBS of the subthalamic nucleus in cervical dystonia and for GPi DBS of secondary dystonias or other focal dystonias (cranial, upper limb). ${ }^{29}$ The full benefit of GPi DBS in dystonia is usually achieved after several months, with stable efficacy for more than 5 years. ${ }^{30,31}$ 


\section{Box 2. Secondary dystonias}

\section{Non-degenerative combined dystonia syndromes}

Dopa-responsive dystonia: results from genetic defects in enzymes that are involved in the biosynthesis of dopamine; the most common condition is autosomal dominant GTP cyclohydrolase 1 deficiency (Segawa disease)

> Rapid onset dystonia-parkinsonism (ATP1A3 gene mutations)

$>$ Myoclonus-dystonia: most common cause is SGCE gene mutations

\section{Dystonia associated with neurodegenerative disorders}

\section{Autosomal dominant}

- Huntington disease

- Machado-Joseph disease (SCA3)

- Basal ganglia calcifications syndromes

- SCAs

- Neuroferritinopathy

\section{Autosomal recessive}

- Juvenile Parkinson's disease

- Wilson's disease

- Neuroacanthocytosis syndromes

- Hallervorden-Spatz disease

- Neurodegeneration with brain iron accumulation syndromes (pantothenate kinase associated neurodegeneration, PLA2G6 associated neurodegeneration, Kufor Rakeb syndrome, MPAN, BPAN, aceruloplasminemia)

- Lysosomal storage disorders

- Ataxia-Telangiectasia

- Homocystinuria

\section{Recessive X-linked}

- Lubag disease

- Lesh-Nyhan syndrome

- Rett syndrome

Mitochondrial disorders

- Leber disease

- MELAS

- MERRF

- Leigh's disease

\section{Parkinsonian syndromes}

- Parkinson's disease

- Progressive Supranuclear Palsy

- Cortico-basal syndrome

- Multiple system atrophy

\section{Dystonia associated with acquired causes}

\section{Medications}

- Dopaminergic (L-dopa, dopamine agonists), dopamine receptor blocking drugs (neuroleptics, prochlorperazine, metoclopramide), selective serotonin reuptake inhibitors, MAO inhibitors, antiepileptic drugs, ergots, flecainide, cocaine, ranitidine, calcium antagonists, anaesthetic agents.

\section{Toxins}

- Manganese, carbon monoxide, carbon disulfide, cyanide, methanol, disulfiram

\section{$>$ Infectious, post-infectious and inflammatory diseases}

- Subacute sclerosing panencephalopathy, Reye's syndrome, viral encephalitis, Creutzfeld-Jakob disease, systemic lupus erythematosus, Antiphospholipid syndrome, Sjogren's syndrome.

\section{CNS lesion}

- Brain tumour, Stroke, Hypoxia, Intracranial haemorrhage, CNS trauma, congenital malformations, cervical cord lesions.

\section{Perinatal cerebral injury}

- Cerebral palsy, delayed-onset dystonia, perinatal hypoxia kernicterus.

\section{Functional dystonia}

BPAN = beta-propeller protein-associated neurodegeneration; $C N S=$ central nervous system; $\mathrm{GTP}=$ guanosine triphosphate; $\mathrm{MAO}=$ monoamine oxydase; $\mathrm{MELAS}=$ mitochondrial encephalomyopathy, lactic acidosis, and stroke-like episodes; MERRF = myoclonic epilepsy with ragged red fibers; $\mathrm{MPAN}=$ mitochondrial membrane protein-associated neurodegeneration; SCAs = spinocerebellar ataxias.

\section{Chorea}

\section{Clinical presentation}

Chorea is a movement disorder characterised by rapid, purposeless, non-stereotyped movements flowing randomly from one part of the body to another. ${ }^{32}$ Chorea is caused by a multitude of conditions with different pathophysiological mechanisms. In the majority of cases, the clinical context in which chorea develops is the key to defining its aetiology. The different causes of chorea may be recognised based on course (acute/subacute, ie reaching a peak of severity within days/ weeks, or chronic, ie gradually progressing over months or years) and body distribution (focal, segmental, unilateral, generalised). An updated list of the different aetiologies of chorea and relative diagnostic work-up is provided in Table S1.

Within the rubric of acute/subacute chorea, the spectrum of immune-mediated causes of chorea has broadened extensively to include a vast group of autoantibody-induced encephalopathies (such as those associated with anti-LGIl, anti-GAD65, anti-CASPR2, anti-GABA-B receptor antibodies, in addition to the choreiform movements documented in anti-NMDA-receptor antibody-related encephalitis). ${ }^{33}$ Furthermore, there is increasing interest in the behavioural and cognitive aspects of the prototype of immune-mediated choreas, ie Sydenham's (or rheumatic) chorea (SC). ${ }^{34}$ Executive dysfunction, obsessive-compulsive disorder, mood disorders, anxiety, ADHD, tic disorders and (rarely) psychosis are important comorbidities in patients with SC. ${ }^{33}$

Among chronic or progressive chorea, Huntington's disease (HD) remains the most frequent cause. This is an autosomal dominant trinucleotide repeat disorder related to the IT15 gene coding for the protein huntingtin. ${ }^{35}$ Usually, chorea is the prominent movement disorder of adult-onset HD, whereas juvenile HD (Westphal variant) typically presents with an akinetic-rigid syndrome and progresses more rapidly. ${ }^{36}$ Personality changes, depression, hostility and/or increased interpersonal sensitivity, obsessive-compulsive symptoms, phobias and anxiety appear early in the course of the illness. As 
the disease progresses, global cognitive decline appears, initially mainly manifesting as loss of cognitive flexibility, executive dysfunction, memory and attention deficits and reduced psychomotor speed. ${ }^{37,38}$

A number of HD lookalikes have been described. Within this group, $c 9$ orf 72 expansions, spanning across all age groups, have recently been described as the most commonly identified genetic cause (1.95\%), followed by spinocerebellar ataxia type 17, Huntington's disease-like 2, Friedreich ataxia, and inherited prion disease (HDL-1). The group of HD phenocopies is in continuous expansion. ${ }^{39}$

Among the forms of chorea beginning in youth and showing a slowly progressive or apparently non-progressive course, there have been recent advances in the characterisation of benign hereditary chorea, which was confirmed to be genetically heterogeneous. This condition is mostly caused by NKX2.1 autosomal dominant mutations, which should be screened for where there is a history of neonatal hypotonia, motor developmental delay, and early-onset hyperkinetic movement disorder (often generalised chorea and/or dystonia) with possible comorbid lung or thyroid symptoms. ${ }^{40}$ Early-onset chorea and dystonia can also be distinguishing features of the recently identified autosomal dominant inherited adenylate cyclase-5 (ADCY5) mutations. ${ }^{41}$

\section{Treatment}

The treatment of chorea aims to reverse treatable conditions, followed or accompanied by symptomatic management. Symptomatic pharmacological management is necessary when chorea is not fully reversible (eg neurodegenerative or vascular causes) or if aetiologic treatment is complex and the patient needs fast and effective improvement. Three main classes of medications are used to alleviate chorea:

1 D2 dopamine receptor blockers (antipsychotics)

2 tetrabenazine, indicated to treat chorea in $\mathrm{HD}$ and tardive choreiform dyskinesias

3 antiepileptic agents (mainly carbamazepine and valproic acid).

In patients with chorea and severe behavioural symptoms (eg psychosis, depression and aggressive behaviour) antipsychotics may be favoured, whereas tetrabenazione may be more difficult to manage in the presence of severe depression with suicidal risk. A combination of antipsychotics and tetrabenazine can be considered, if safe, when monotherapy is unsuccessful. Antiepileptic drugs like carbamazepine and valproate are less effective anti-chorea agents, with the exception of paroxysmal kinesigenic chorea, which responds dramatically to carbamazepine. $^{42,43}$

Surgical treatment (either ablative or DBS) of chorea is still at an experimental level and it should be used in select cases refractory to pharmacological treatments. Evidence supporting surgical treatment of chorea is quite limited and focuses mainly on HD and neuroacanthocytosis syndromes. ${ }^{44}$ The GPi is the target of deep brain stimulation for relieving chorea. However, long-term outcomes are still uncertain, considering the progressive course of these conditions. DBS of the GPi in tardive choreiform dyskinesias is supported by recent promising evidence. ${ }^{45}$

\section{Conclusion}

Because of the wide variety of different aetiological causes of movement disorders and a large number of different forms of clinical presentation, it is important to use a step-by-step organised approach to patients with a movement disorder. The first step is to identify whether it is a hypo- or hyperkinetic movement disorder and, if the latter, which particular form of dyskinesia. Age and distribution and whether there are associated other signs or clinical features is to be noted.

\section{Supplementary material}

Additional supplementary material may be found in the online version of this article at www.clinmed.rcpjournal.org/:

S1 - Clinical approach to chorea

\section{Conflicts of interests}

The authors declare no conflicts of interest.

\section{References}

1 Abdo WF, van de Warrenburg BP, Burn DJ, Quinn NP, Bloem BR. The clinical approach to movement disorders. Nat Rev Neurol 2010;6:29-37.

2 American Psychiatric Association. Diagnostic and statistical manual of mental disorders, 5th edn. Arlington, VA: American Psychiatric Publishing, 2013.

3 Ganos C, Martino D. Tics and Tourette syndrome. Neurol Clin 2015;33:115-36.

4 Knight T, Steeves T, Day L et al. Prevalence of tic disorders: a systematic review and meta-analysis. Pediatr Neurol 2012;47:77-90.

5 Hirschtritt ME, Lee PC, Pauls DL et al. Lifetime prevalence, age of risk, and genetic relationships of comorbid psychiatric disorders in Tourette syndrome. JAMA Psychiatry 2015;72:325-33.

6 Serrien DJ, Orth M, Evans AH, Lees AJ, Brown P. Motor inhibition in patients with Gilles de la Tourette syndrome: functional activation patterns as revealed by EEG coherence. Brain 2005;128:116-25.

7 Worbe Y, Marrakchi-Kacem L, Lecomte S et al. Altered structural connectivity of cortico-striato-pallido-thalamic networks in Gilles de la Tourette syndrome. Brain 2015;138:472-82.

8 Niccolai V, van Dijk H, Franzkowiak S et al. Increased beta rhythm as an indicator of inhibitory mechanisms in Tourette syndrome. Mov Disord 2015;31:384-92.

9 McCairn KW, Nagai Y, Hori Y et al. A primary role for nucleus accumbens and related limbic network in vocal tics. Neuron 2016;89:300-7.

10 Kataoka Y, Kalanithi PS, Grantz H et al. Decreased number of parvalbumin and cholinergic interneurons in the striatum of individuals with Tourette syndrome. J Comp Neurol 2010;518:277-91.

11 Roessner V, Plessen KJ, Rothenberger A et al. European clinical guidelines for Tourette syndrome and other tic disorders. Part II: pharmacological treatment. Eur Child Adolesc Psychiatry 2011;20:173-96.

12 McGuire JF, Piacentini J, Brennan EA et al. A meta-analysis of behavior therapy for Tourette syndrome. J Psychiatr Res 2014;50:106-12.

13 Wile DJ, Pringsheim TM. Behavior therapy for Tourette syndrome: a systematic review and meta-analysis. Curr Treat Options Neurol 2013;15:385-95.

14 Weisman H, Qureshi IA, Leckman JF, Scahill L, Bloch MH. Systematic review: pharmacological treatment of tic disorders-efficacy of antipsychotic and alpha-2 adrenergic agonist agents. Neurosci Biobehav Rev 2013;37:1162-71.

15 Mogwitz S, Buse J, Ehrlich S, Roessner V. Clinical pharmacology of dopamine-modulating agents in Tourette's syndrome. Int Rev Neurobiol 2013;112:281-349. 
16 Schrock LE, Mink JW, Woods DW et al. Tourette syndrome deep brain stimulation: review and updated recommendations. Mov Disord 2015;30:448-71.

17 Kefalopoulou Z, Zrinzo L, Jahanshahi M et al. Bilateral globus pallidus stimulation for severe Tourette's syndrome: a double-blind, randomised crossover trial. Lancet Neurol 2015;14:595-605.

18 Ackermans L, Duits A, van der Linden C et al. Double-blind clinical trial of thalamic stimulation in patients with Tourette syndrome. Brain 2011;134:832-44.

19 Geyer HL, Bressman SB. The diagnosis of dystonia. Lancet Neurol 2006;5:780-90.

20 Albanese A, Lalli S. Is this dystonia? Mov Disord 2009;24:1725-31.

21 Fung VS, Jinnah HA, Bhatia K, Vidailhet M. Assessment of patients with isolated or combined dystonia: an update on dystonia syndromes. Mov Disord 2013;28:889-98.

22 Martino D, Liuzzi D, Macerollo A et al. The phenomenology of the geste antagoniste in primary blepharospasm and cervical dystonia. Mov Disord 2010;25:407-12.

23 Albanese A, Bhatia K, Bressman SB et al. Phenomenology and classification of dystonia: a consensus update. Mov Disord 2013;28:863-73.

24 B1 Balint, Bhatia KP. Dystonia: an update on phenomenology, classification, pathogenesis and treatment. Curr Opin Neurol 2014;27:468-76.

25 Albanese A, Barnes MP, Bhatia KP et al. A systematic review on the diagnosis and treatment of primary (idiopathic) dystonia and dystonia plus syndromes: report of an EFNS/MDS-ES Task Force. Eur J Neurol 2006;13:433-44.

26 Jankovic J. Treatment of dystonia. Lancet Neurol 2006;5:864-72.

27 Simpson DM, Blitzer A, Brashear A et al. Assessment: Botulinum neurotoxin for the treatment of movement disorders (an evidencebased review): report of the Therapeutics and Technology Assessment Subcommittee of the American Academy of Neurology. Neurology 2008;70:1699-706.

28 Albanese A, Asmus F, Bhatia KP et al. EFNS guidelines on diagnosis and treatment of primary dystonias. Eur J Neurol 2011;18:5-18.

29 Volkmann J, Wolters A, Kupsch A et al. Pallidal deep brain stimulation in patients with primary generalised or segmental dystonia: 5-year follow-up of a randomised trial. Lancet Neurol 2012;11:1029-38.

30 Vidailhet M, Vercueil L, Houeto JL et al. Bilateral deep-brain stimulation of the globus pallidus in primary generalised dystonia. N Engl J Med 2005;352:459-500.
31 Tierney TS, Lozano AM. Surgical treatment for secondary dystonia. Mov Disord 2012;27:1598-605.

32 L1 Burnett, Jankovic J. Chorea and ballism. Curr Opin Neurol Neurosurg 1992;5:308-13.

33 O'Toole O, Lennon VA, Ahlskog JE et al. Autoimmune chorea in adults. Neurology 2013;80:1133-44.

34 Williams KA, Swedo SE. Post-infectious autoimmune disorders: Sydenham's chorea, PANDAS and beyond. Brain Res 2015;1617:144-54.

35 MacDonald ME, Abrose CM, Duyao MP et al. A novel gene containing a trinucleotide repeat that is expanded and unstable on Huntington's disease chromosomes. Cell 1993;72:971-83.

36 Quarrell OW, Nance MA, Nopoulos P et al. Managing juvenile Huntington's disease. Neurodegener Dis Manag 2013;3:267-76.

37 Paulsen JS and Conybeare RA. Cognitive changes in Huntington's disease. Adv Neurol 2005:96:209-25.

38 Morreale MK. Huntington's disease: looking beyond the movement disorder. Adv Psychosom Med 2015;34:135-42.

39 Martino D, Stamelou M, Bhatia KP. The differential diagnosis of Huntington's disease-like syndromes: 'red flags' for the clinician. J Neurol Neurosurg Psychiatry 2013;84:650-66.

40 KJ1 Peall, Lumsden D, Kneen R et al. Benign hereditary chorea related to NKX2.1: expansion of the genotypic and phenotypic spectrum. Dev Med Child Neurol 2014;56:642-8.

41 Mencacci NE, Erro R, Wiethoff S et al. ADCY5 mutations are another cause of benign hereditary chorea. Neurology 2015;85:80-8.

42 Bhatia KP. Paroxysmal dyskinesias. Mov Disord 2011;26:1157-65.

43 Jankovic J. Treatment of hyperkinetic movement disorders. Lancet Neurol 2009;8:844-56.

44 Edwards TC, Zrinzo L, Limousin P, Foltynie T. Deep brain stimulation in the treatment of chorea. Mov Disord 2012;27:357-63.

45 Pouclet-Courtemanche H, Rouaud T, Thobois S et al. Long-term efficacy and tolerability of bilateral pallidal stimulation to treat tardive dyskinesia. Neurology 2016;86:651-9.

\section{Address for correspondence: Dr D Martino, International Parkinson's Centre of Excellence, King's College and King's College Hospital, Denmark Hill Campus, London SE5 9RS, UK. Email: davidemartino@nhs.net}

